\title{
An inversion technique for the calculation of embedding potentials
}

\author{
O. Roncero, ${ }^{1, a)}$ M. P. de Lara-Castells, ${ }^{1}$ P. Villarreal, ${ }^{1}$ F. Flores,${ }^{2}$ J. Ortega,${ }^{2}$ M. Paniagua,${ }^{3}$ \\ and A. Aguado ${ }^{3}$ \\ ${ }^{1}$ Instituto de Física Fundamental, CSIC, Unidad Asociada UAM-CSIC, Serrano 123, 28006 Madrid, Spain \\ ${ }^{2}$ Departamento de Física Teórica de la Materia Condensada, Facultad de Ciencias C-V, \\ Universidad Autónoma de Madrid, 28049 Madrid, Spain \\ ${ }^{3}$ Unidad Asociada UAM-CSIC, Departamento de Química Física, Facultad de Ciencias C-XIV, \\ Universidad Autónoma de Madrid, 28049 Madrid, Spain
}

(Received 3 July 2008; accepted 3 October 2008; published online 10 November 2008)

\begin{abstract}
A new embedding method to include local correlation in large systems is proposed. In this method the density of the whole system, calculated via density functional theory approaches, is partitioned in two pieces, one corresponding to the subsystem of interest and the rest to the environment. In the second step, an embedding potential is obtained iteratively using as a driving force the self-repulsion due to the density difference, in a similar form as proposed by Zhao et al. [Phys. Rev. A 50, 2138 (1994)], to obtain the "exact" exchange-correlation functional. Such potential is added to the Fock equation to build the localized molecular orbitals which are further used to include the local electronic correlation in the subsystem of interest. This method is an alternative to the previous DFT-based embedding methods first proposed by Wesolowski and Washell [J. Phys. Chem. 97, 8050 (1993)] and after enhanced by Govind et al. [J. Chem. Phys. 110, 7677 (1999)] and adapted to metal extended systems, which use density functionals to describe the kinetic energy contribution to the embedding potential, whose precise form has been largely treated in the literature and its crucial role is discussed here. The method is applied to hydrogen chains and its van der Waals interaction with $\mathrm{H}_{2}$. The results obtained are in very good agreement with exact calculations performed on the whole system, which demonstrates that the method proposed is a very promising route to introduce correlation in large systems. (C) 2008 American Institute of Physics.
\end{abstract}

[DOI: $10.1063 / 1.3007987]$

\section{INTRODUCTION}

Density functional theory (DFT) is nowadays the most widely used method for electronic structure calculations on extended systems. For example, the reactivity of simple molecules on some metals compares nicely with experimental results when using gradient corrected DFT techniques to describe the potential energy surface. ${ }^{1}$ DFT methods have also been widely used to study the reactivity on metal clusters, and recent studies of the reaction of $\mathrm{H}_{2}$ on metal clusters ${ }^{2,3}$ have shown that the high dissociation barrier for ordered clusters is dramatically reduced when the cluster gets distorted. Local density approximation works well for homogeneous electron densities, typically associated with metals, but it has difficulties in describing nonhomogeneous densities, such as those associated with insulators or distorted metallic clusters. Besides its great success, it presents several disadvantages, especially when dealing with open-shell species, excited electronic states, van der Waals interactions, etc. Although tremendous improvements in the functionals have been made, for example, in generalized gradient corrected approximations, it is desirable to consider wave function based methods, which can handle electronic correlation in a systematic way.

Important advances have been made in local correlation methods for large systems ${ }^{4,5}$ and in the increment method for

${ }^{a)}$ Electronic mail: oroncero@imaff.cfmac.csic.es. solids. ${ }^{6-9}$ These kind of methods use localized orbitals ${ }^{10-12}$ or the equivalent Wannier orbitals for periodic systems ${ }^{13,14}$ because the correlation can be considered local, i.e., the Hamiltonian matrix elements decreases as the "average distance" between the localized orbitals involved increases, yielding a significant reduction in the number of matrix elements in this representation. These local orbitals, $\phi_{l}$, are obtained by linear transformation from the Hartree-Fock (HF) orbitals ${ }^{10-12}$ and are not eigenvalues of the Fock operator $\hat{\mathcal{F}}$. However, they are solutions of a modified HF equation of the form ${ }^{10}$

$$
[\hat{\mathcal{F}}+\hat{\mathcal{V}}] \phi_{l}=\epsilon_{l} \phi_{l}
$$

where $\hat{\mathcal{V}}$ is a suitably defined operator, which plays a role formally analogous to the correlation potential in DFT. In the localized representation, the HF solution is no longer single determinant, and this is why typically only virtual orbitals are localized. These methods are computationally cheaper than the usual highly correlated ab initio methods, but they are nevertheless too demanding.

A possible alternative consists in localizing the density, dividing the system into pieces, determining the electronic density for each of them separately. These partial densities are then used to reconstruct the total density of the system. One of the former methods within this framework is the socalled embedded atoms-in-molecules DFT approach applied for molecules ${ }^{15-20}$ and solids. ${ }^{21-24}$ Such methods are based 
on the reconstruction of the first-order density matrix of the whole system by the simple addition of the densities associated with each of the subsystems, imposing the $N$-representability conditions. ${ }^{25,26}$

A natural extension of such work consists in including the correlation by calculating each of the second-order density matrices and then reconstructing that of the whole system, as done lately in the buffered divide-and-conquer techniques. ${ }^{27-29}$ Even when these methods are very promising, they have the problem of a nonunique "purification" procedure to obtain a reconstructed second-order density matrix, which fulfills the required spin and $N$-representability conditions. $^{30,31}$

In most of the DFT-based embedded approaches the density of a subsystem is obtained iteratively using the mean field potential of the rest of the whole system or environment, hereafter called "embedding" potential. ${ }^{32}$ In the conventional cluster approach, the whole system is separated into the primary region, formed by the "cluster" of atoms where the process under study occurs, and the rest of the system or environment. The use of standard $a b$ initio methods on the cluster, adding some atoms at its edges to saturate bonds, can yield good results in covalent insulating systems. ${ }^{33-36}$ In ionic crystals, the cluster must be embedded in the background formed by the charges in the surroundings, described by core pseudopotentials, for cations or point charges for anions and account for long-range interactions. ${ }^{37-40}$ The embedding potentials calculated using these embedding DFT techniques provides a natural improvement to describe the interaction of a subsystem and its environment. $^{22,41}$

A further refinement in the embedded DFT approach has been recently developed by Carter and co-workers ${ }^{42-44}$ to treat metals whose delocalized electronic density complicates any factorization. In this method, the density of the whole system and that of the "active" cluster are first obtained from DFT calculations, and the embedding potential is derived from them iteratively following Wesolowski and Warshel's method. ${ }^{32}$ The embedding potential is then added to the HF equation, as in Eq. (1), to obtain the molecular orbitals and with them any conventional $a b$ initio calculation can be performed, including electron correlation effects, opening the possibility of calculating excited electronic states.

As noted by Cortona, ${ }^{23}$ the nonadditivity of the kinetic energy functionals in DFT creates a repulsive term surrounding the cluster in the embedding potential, of crucial importance in maintaining localized the electronic density in the cluster. One of the difficulties of Wesolowski and Warshel's ${ }^{32}$ method is related to the kinetic energy functional, and several of them have been used ${ }^{42-44}$ and have been the subject of many studies. ${ }^{45,46}$ In DFT formalism, this problem is somehow overcame using the Kohn-Sham equation but it presents an analogous problem, the determination of the exchangecorrelation potential. In this direction, an intense field of research in this area consists in developing methods to extract (or "invert") the exchange-correlation potential from the electronic density obtained with an "exact" method. ${ }^{47-57} \mathrm{~A}$ very promising evolution has been recently addressed in the so-called direct optimized effective potential ${ }^{58-64}$ or $a b$ initio DFT $^{65,66}$ methods, in which the exchange potential is treated exactly and the formal expression of the correlation potential is numerically obtained in a second-order perturbation treatment of the electron-electron interaction.

In this work we present an alternative embedding method based on the analogy played by the embedding potential in the modified HF equation, Eq. (1), and the correlation potential using the Hartree-Fock-Kohn-Sham equation. ${ }^{67-70}$ In this method, first the density of the whole system is divided into two parts, one describing the active cluster, $\rho_{A}$, and the other the rest of the system, $\rho_{B}$, in a similar way as is done in the atoms-in-molecule DFT procedure. ${ }^{18}$ Several criteria for such a dividing procedure can be followed, such as minimizing the interaction of the two electron densities obtained. In this regard, there is no restriction about the method employed to obtain the total density, even when, for large systems, a DFT method is commonly used. In the second step, $\rho_{A}$ is used to invert the embedding potential in a HF equation by minimizing the self-interaction in a procedure similar to that proposed by Zhao et al. $^{50}$ [hereafter called the Zhao-Morrison-Parr (ZMP) procedure]. The embedding potential thus obtained is approximated unless the $v$ representability of $\rho_{A}$ is imposed during the partition procedure of the density of the whole system. Finally, once the embedding potential $\mathcal{V}$ is known, $a b$ initio calculations at the desired level of accuracy are performed for the ground and excited electronic states.

The method is applied to chains of hydrogen atoms and the results are compared with exact results. The paper is organized as follows. First, the role played by the kinetic energy is analyzed in Sec. II by comparing a HF treatment with the DFT embedding method from Wesolowski and Warshell $^{32}$ and by examining the barriers appearing in the edges of the cluster, as described by Cortona. ${ }^{23}$ In Sec. III, the new embedding method proposed here is described, whereas the results obtained for hydrogen chains on the ground state are presented and discussed in Sec. IV. Finally, Sec. V is devoted to some conclusions.

\section{THE KINETIC ENERGY IN EMBEDDING PROCEDURES}

Let us consider a closed-shell system formed by $n$ electrons and $N$ nuclei whose electronic Hamiltonian is expressed as

$$
H=H^{A}+H^{B}+V^{A B}+W^{A B},
$$

with

$$
H^{C}=\sum_{i \in C}\left\{T_{i}+V^{C}\left(\mathbf{r}_{\mathbf{i}}\right)+W^{C}\left(\mathbf{r}_{\mathbf{i}}, \mathbf{r}_{\mathbf{j}}\right)\right\}
$$

where $C=A$ or $B$, and the kinetic term reads

$$
T_{i}=-\frac{\hbar^{2}}{2 m} \nabla_{i}^{2},
$$

while nuclear potential terms are given by 


$$
V^{C}\left(\mathbf{r}_{\mathbf{i}}\right)=-\sum_{a \in C} \frac{Z_{a}}{r_{i a}}+\sum_{a \in C}^{N} \sum_{b>a \in C}^{N} \frac{Z_{a} Z_{b}}{R_{a b}}
$$

and the electronic repulsion is

$$
W^{C}\left(\mathbf{r}_{\mathbf{i}}, \mathbf{r}_{\mathbf{j}}\right)=\sum_{j>i \in C} \frac{1}{r_{i j}} .
$$

In these expressions the sums run over nuclei and electrons on each subsystem $A$ or $B$, i.e., $i \in A \rightarrow i=1, \ldots, n^{A}$, $a \in A \rightarrow a=1, \ldots, N^{A}$ for $C=A$ and $i \in B \rightarrow i=n^{A}+1, \ldots, n$, $a \in B \rightarrow a=N^{A}+1, \ldots, N^{A}$ for $C=B$. For simplicity, it will also be assumed that $A$ and $B$ correspond to closed-shell subsystems. In Eq. (2), the coupling terms are given by

$$
\begin{aligned}
V^{A B} & =-\sum_{a \in B} \sum_{i \in A} \frac{Z_{a}}{r_{i a}}-\sum_{a \in A} \sum_{i \in B} \frac{Z_{a}}{r_{i a}}+\sum_{a \in A} \sum_{b \in B} \frac{Z_{a} Z_{b}}{R_{a b}}, \\
W^{A B} & =\sum_{i \in A} \sum_{j \in B} \frac{1}{r_{i j}},
\end{aligned}
$$

with $V=V^{A}+V^{B}+V^{A B}$ and $W=W^{A}+W^{B}+W^{A B}$ being the nuclear attraction and electronic repulsion interactions of the whole system, respectively. The monoelectronic operators thus defined are represented in a basis set of atomic functions centered on each nucleus, $|\nu\rangle$, giving rise to the $\mathbf{H}_{0}=\mathbf{T}+\mathbf{V}$ matrices, the core, kinetic, and nuclear attraction matrices (if a superscript $A$ or $B$ is added such matrices refer to one of the two subsystems defined). The bielectronic term represented in this basis gives rise to the usual Coulomb and exchange operators, whose matrix elements are

$$
\begin{gathered}
J_{\nu \mu}=\frac{1}{2} \sum_{\gamma \delta} \rho_{\gamma \delta}\langle\nu \gamma \| \mu \delta\rangle, \\
\left.K_{\nu \mu}=\frac{1}{2} \sum_{\gamma \delta} \rho_{\gamma \delta} \delta \gamma \nu \| \mu \delta\right\rangle .
\end{gathered}
$$

In these expressions, the matrix elements of the ground-state first-order density matrix have been used, so that

$$
\rho(\mathbf{r})=\sum_{\nu, \mu}\langle\mathbf{r} \mid \nu\rangle \rho_{\nu \mu}\langle\mu \mid \mathbf{r}\rangle .
$$

The first-order density matrix is factorized as

$$
\rho=\rho^{A}+\rho^{B}
$$

and it is assumed that $\rho^{A}$ and $\rho^{B}$ may overlap.

These two densities do not correspond to the densities of the isolated subsystems $A$ and $B$. However, $\rho^{A}(\mathbf{r})$ and $\rho^{B}(\mathbf{r})$ can be associated with the ground eigenstates of the corresponding modified monoelectronic Hamiltonian of each of the two subsystems, given by

$$
\mathcal{H}^{C}=H^{C}+\mathcal{V}^{C},
$$

with eigenvalues $\mathcal{E}^{C}$, where the so-called "embedding potentials", $\mathcal{V}^{C}$, are chosen to yield, for each subsystem, the charges $\rho^{A}(\mathbf{r})$ or $\rho^{B}(\mathbf{r})$. Below, the equations for DFT methods will be discussed, but they are immediately applicable to
HF method by substituting the exchange-correlation potential by the HF exchange term.

\section{A. DFT approach}

The energy of the system is a functional of the electronic density for the ground state given by ${ }^{71}$

$$
E[\rho]=T_{s}[\rho]+J[\rho]+E_{\mathrm{xc}}[\rho]+\int d \mathbf{r} V(\mathbf{r}) \rho(\mathbf{r}),
$$

where $T_{s}$ is the noninteracting kinetic energy functional, $J$ is the Coulomb interaction potential, $V(\mathbf{r})$ is the external potential due to the nuclear attraction, while $E_{\mathrm{xc}}$ is the exchangecorrelation functional. Since the explicit form of $E_{\mathrm{xc}}$ is not known, approximate functionals are used.

Similar expressions hold for the energies associated with each of subsystems $A$ and $B$ but modified with the inclusion of the embedding potential as

$$
\begin{aligned}
\mathcal{E}^{C}[\rho]= & T_{s}\left[\rho^{C}\right]+J\left[\rho^{C}\right]+E_{\mathrm{xc}}\left[\rho^{C}\right]+\int d \mathbf{r}[V(\mathbf{r}) \\
& \left.+\mathcal{V}^{C}(\mathbf{r})\right] \rho^{C}(\mathbf{r}) .
\end{aligned}
$$

We can now make use of the Hohenberg-Kohn identities

$$
\frac{\delta E[\rho(\mathbf{r})]}{\delta \rho(\mathbf{r})}=\mu \quad \text { and } \quad \frac{\delta E^{C}\left[\rho^{C}(\mathbf{r})\right]}{\delta \rho^{C}(\mathbf{r})}=\mu,
$$

where the $\mu$ is the chemical potential of the system, to conclude that

$$
\frac{\delta E\left[\rho(r)=\rho^{A}(\mathbf{r})+\rho^{B}(\mathbf{r})\right]}{\delta \rho^{A}(\mathbf{r})}=\frac{\delta E^{A}\left[\rho^{A}(\mathbf{r})\right]}{\delta \rho^{A}(\mathbf{r})},
$$

where we have introduced charge variations such that $\delta \rho$ $=\delta \rho^{A}$ and $\delta \rho^{B}=0$, since $\rho=\rho^{A}+\rho^{B}$. This allows us to conclude that ${ }^{42-44}$

$$
\begin{aligned}
\mathcal{V}^{A}= & \frac{\delta T_{S}[\rho]}{\delta \rho}-\frac{\delta T_{S}\left[\rho^{A}\right]}{\delta \rho^{A}}+\frac{\delta J[\rho]}{\delta \rho}-\frac{\delta J\left[\rho^{A}\right]}{\delta \rho^{A}}+\frac{\delta V_{\mathrm{xc}}[\rho]}{\delta \rho} \\
& -\frac{\delta V_{\mathrm{xc}}\left[\rho^{A}\right]}{\delta \rho^{A}}+V^{B} .
\end{aligned}
$$

Different functions can be assumed to describe the exchange-correlation term. ${ }^{32,41-44}$ The important point is the nonlinearity of such functionals, as first noted by Cortona ${ }^{23}$ and thereafter studied by many authors: ${ }^{45,54,55,72}$ they introduced a repulsive potential in the edges of the cluster describing subsystem $A$, thus avoiding the delocalization of the electronic density out of region $A$.

To illustrate such an effect we shall consider the simple Thomas-Fermi kinetic energy functional, $T_{T F}[\rho]=C \int d \mathbf{r} \rho^{5 / 3}$, applied to a diatomic model system represented by the electronic densities $\rho(r)=\left|a e^{-\alpha r_{A}^{2}}+b e^{-\beta r_{B}^{2}}\right|^{2}$ and $\rho^{A}(r)=\left|a^{\prime} e^{-\alpha r_{A}^{2}}\right|^{2}$. Using the Thomas-Fermi functional, the first two terms in Eq. (14) take the form

$$
\mathcal{V}_{T}^{A} \propto\left(a e^{-\alpha r_{A}^{2}}+b e^{-\beta r_{B}^{2}}\right)^{4 / 3}-\left(a^{\prime} e^{-\alpha r_{A}^{2}}\right)^{4 / 3} .
$$

An example is shown in Fig. 1 for a particular set of parameters. It is clearly seen that the kinetic contribution to the embedding potential for atom $A$ presents a repulsive barrier 


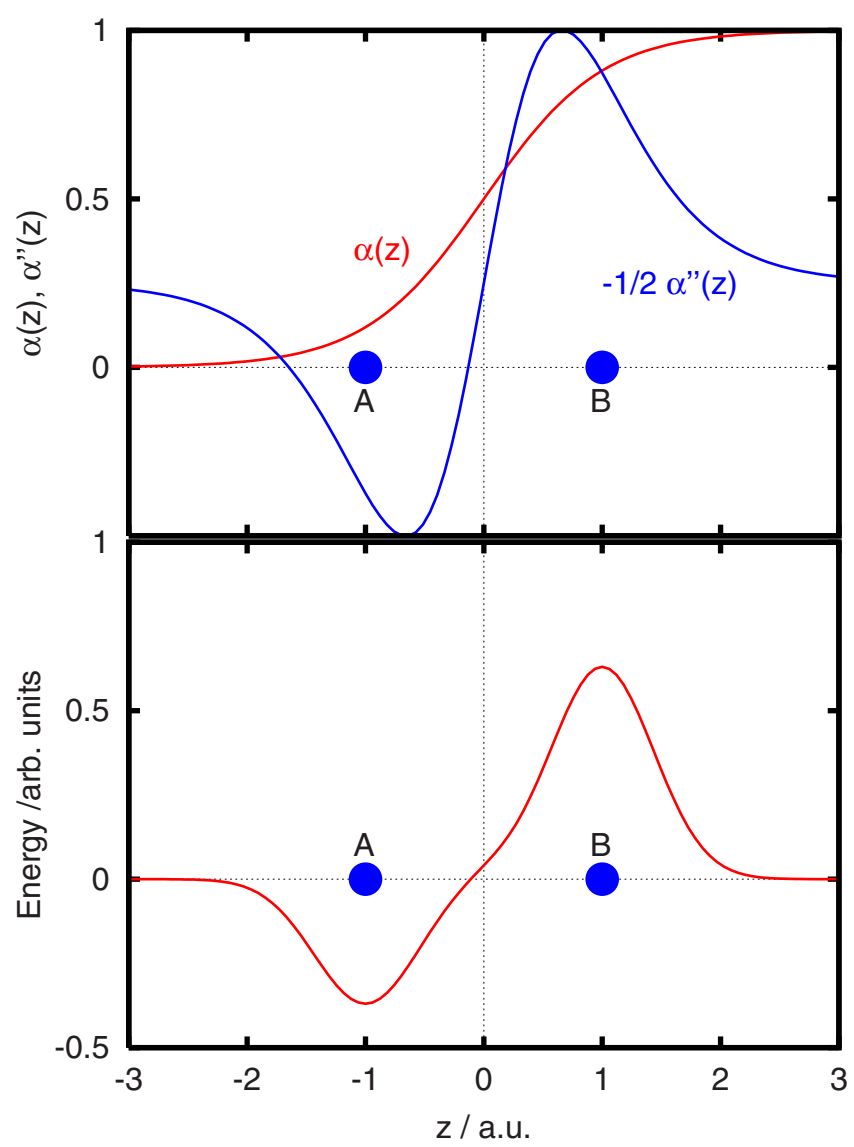

FIG. 1. (Color online) Bottom panel: kinetic energy contribution to the embedding potential $\mathcal{V}_{T}^{A}$ corresponding to the first two terms in Eq. (14), corresponding to the model system explained in the text for $A$ and $B$ being located along the $z$ axis at -1 and 1 , respectively, with $\alpha=\beta=2, a=b=1 / \sqrt{(2)}$, and $a^{\prime}=1$. Top panel: the dividing function $\alpha(r)=[1+\tanh (\gamma z)] / 2$ and $-\frac{1}{2} \alpha^{\prime \prime}(z)$ are shown.

around atom $B$ while producing a well in the region of $A .^{17}$ Thus, such term introduces a "force" in the system that localizes the $\rho^{A}$ density around atom $A$.

The embedding potential in Eq. (14) is obtained selfconsistently, starting from the densities of the whole system and that of subsystem $A$. If the kinetic energy terms in Eq. (14) are removed, the iterative density associated with $A$, initially located in fragment $A$, tends to delocalize in the whole system as the iteration proceeds, yielding molecular orbitals delocalized in the whole system, as it occurs in conventional HF calculations. The repulsive potentials introduced by the kinetic terms are then crucial to separate the densities between the different subsystems in any embedding method, its origin being in the nonlinearity of the kinetic energy functionals. Because of its importance, it is worthwhile to analyze further its role.

\section{B. The localization barrier}

For this purpose, it is interesting to note the similarity played by the embedding potential thus obtained and the $\mathcal{V}$ term in the modified HF equation, Eq. (1), whose solutions are localized orbitals as reported by Edmiston and Ruedenberg. ${ }^{10}$ To get this additional term of the localization equation, we start by partitioning the molecular orbitals $\phi^{k}$ as

$$
\phi^{k}=\alpha \phi^{k}+\beta \phi^{k}=\phi_{A}^{k}+\phi_{B}^{k},
$$

where $\alpha(r)$ is a function chosen such that it "projects" in subsystem $A$ according to different criteria, which will be commented below, fulfilling $0<\alpha(r)<1$ and $\beta(r)=1-\alpha(r)$. This partition is then used in the Fock equation to find the effective equation satisfied by $\phi_{A}^{k}$. Local and nonlocal terms can be distinguished. The potential term is local and $V \alpha(\mathbf{r}) \phi^{k}=\alpha(\mathbf{r}) V \phi^{k}$, so that when applied to a localized function it remains localized. The kinetic term, however, is nonlocal and when applied to localized functions it results in

$$
\begin{aligned}
-\frac{1}{2} \boldsymbol{\nabla}^{2} \alpha(\mathbf{r}) \phi^{k}(\mathbf{r})= & -\frac{1}{2} \alpha(\mathbf{r})\left(\boldsymbol{\nabla}^{2} \phi^{k}(\mathbf{r})\right)-\frac{1}{2} \phi^{k}(\mathbf{r})\left(\boldsymbol{\nabla}^{2} \alpha(\mathbf{r})\right) \\
& -(\boldsymbol{\nabla} \alpha(\mathbf{r})) \cdot\left(\boldsymbol{\nabla} \phi^{k}(\mathbf{r})\right),
\end{aligned}
$$

where the two last terms in the right hand side produce a localization, especially when applied iteratively. A very crude approximation to avoid such delocalization would be to eliminate these two terms, which could be achieved by adding a kinetic contribution, as

$$
\mathcal{V}_{T} \propto-\frac{1}{2}\left(\nabla^{2} \alpha(\mathbf{r})\right)-(\boldsymbol{\nabla} \alpha(\mathbf{r})) \cdot \boldsymbol{\nabla},
$$

to the modified localized HF equation, Eq. (1), in a similar way as proposed by Edmiston and Ruedenberg. ${ }^{10} \mathcal{V}_{T}$ is formed by a potential-like term plus a first derivative coupling term, similar in spirit to nonadiabatic derivative couplings. The correction proposed is by far too simple, but it may help in understanding some of the effects we want to stress in this work.

To illustrate the effect of the first term of this $\mathcal{V}_{T}$, which would play a role similar to the kinetic contribution of the embedding potential in Sec. II A, we shall consider a similar model system: two atoms $A$ and $B$ using a projection function $\alpha(r)=[1+\tanh (\gamma z)] / 2$ along the internuclear distance $z$. $\alpha(\mathbf{r})$, and $\left(\nabla^{2} \alpha(\mathbf{r})\right)$, shown in the top panel of Fig. 1 for a particular monodimensional case. It is clearly shown that such term behaves qualitatively in a very similar way to the $\mathcal{V}_{T}^{A}$ term obtained in Wesolowski and Warshell ${ }^{32}$ DFT embedding formalism above.

Even when the correction proposed here is by far too crude and should only be taken on a qualitative basis, it already indicates that designing an effective equation for producing localized orbitals, in the spirit of Edmiston and Ruedenberg, ${ }^{10}$ is somehow analogous to introducing an embedding potential to localize the density in a subsystem $A$ with the proper boundary conditions. In such scenario, the nonlocal (or nonadditive) terms and, in particular, the kinetic energy introduce a barrier in the edges of the localization/ partition region. In addition, some other terms, such as those proportional to first-order derivatives, may be needed to account for the separation of the density. Since the shape of the embedding potential at the edges of the cluster is going to establish the boundary conditions, which are important to connect the cluster with its environment, it should be determined as accurately as possible. The repulsive potential appearing in all DFT-based embedding models, such as Wesolowski and Warshell ${ }^{32}$ method illustrated above, is based on the use of some approximate kinetic energy functional, and 
hence it is desirable to design alternative methods, improving the description of the kinetic energy distribution, such as the one introduced in Sec. III.

\section{THE INVERSION TECHNIQUE FOR THE EMBEDDING POTENTIAL}

In most of DFT-based embedding methods discussed above $^{32,41-44}$ the embedding potential is determined iteratively at the same time as the density of the cluster. In this work we proposed a different strategy, similar to that initially proposed in the DFT atoms-in-molecules techniques, ${ }^{17-19}$ consisting in three consecutive steps:

(1) Determination of the total density $\rho$ (corresponding to the ground electronic state of energy $\left.E_{\text {tot }}\right)$ and determination of a partition or projection function $\alpha(\mathbf{r})$ to obtain $\rho_{A}$, such that $\int d \mathbf{r} \rho_{A}(\mathbf{r})=n_{A}$, with $n_{A}$ being an integer number.

(2) Determination, or "inversion, of $\mathcal{V}$. Defining $\rho_{A}^{k}$ as the density resulting from the solution of the modified HF equation (1), $\mathcal{V}$ is determined by minimizing the $\rho_{A}-\rho_{A}^{k}$ difference, taking $\rho_{A}$ obtained in point (1) as the reference density.

(3) With the embedding potential thus determined, the molecular orbitals are obtained by solving Eq. (1). These orbitals are used to build different electronic configurations in which the electronic Hamiltonian is represented and diagonalized to introduce electronic correlation with $a b$ initio methods [such as multiconfigurational self-consistent field (MCSCF) and/or multireference configuration interaction (MRCI)].

These steps will described separately in more detail below. This procedure has several advantages:

- It does not explicitly use any particular functional for the kinetic energy term, thus avoiding a priori approximations as those discussed for the DFT-based embedding methods described above.

- The total density $\rho$ can be calculated at any ab initio level. For large systems, DFT calculations are expected to provide $\rho$, but there is no a priori for not choosing a more correlated method, which is expected to yield better results.

- This method links the problems of determining embedding and exchange-correlation potentials, thus allowing to join efforts.

The major disadvantages of the method arise in the inversion step (2) of the procedure, because it is not a simple numerical problem.

\section{A. Density partitioning}

The partitioning of the density of a system is very frequently done for various purposes, such as population analysis to build electrostatic models, to rationalize chemical reactivity or describe long-range electrostatic potentials, ${ }^{73}$ and to separate a system in atoms to make more affordable the calculation of the whole system, like in atoms-in-molecule methods. ${ }^{15-24}$ An extension of this last approach consists in obtaining the density of chemically meaningful fragments $^{74,75}$ to recombine them into new molecules. ${ }^{76}$ Since any partition of the electronic density is intrinsically arbitrary, there are a great variety of methods. ${ }^{15,77-80}$

One possibility to determine the partition function $\alpha(r)$ is the minimization of the Coulomb-exchange energy difference $^{18}$

$$
2 J[\rho]-K[\rho]-2 J_{A}\left[\rho_{A}\right]+K_{A}\left[\rho_{A}\right]-2 J_{B}\left[\rho_{B}\right]+K_{B}\left[\rho_{B}\right],
$$

which is equivalent to minimizing nondiagonal Coulombexchange terms between $A$ and $B$.

Being a difficult task, in many cases such partition produces results similar to Mulliken's method, which assigns a half-to-half criterion. Representing the density in an atomic basis, as in Eq. (8), a Mulliken-type partition is

$$
\begin{aligned}
& \rho_{\mu \nu}=\left(P_{\mu \nu}^{A}+P_{\mu \nu}^{B}\right) \rho_{\mu \nu} \\
& \text { with } P_{\mu \nu}^{C}=\left\{\begin{array}{lll}
1 & \text { if } \mu \in C, & \nu \in C \\
1 / 2 & \text { if } \mu \in C, & \nu \notin C \\
0 & \text { if } \mu \notin C, & \nu \notin C .
\end{array}\right.
\end{aligned}
$$

Such expression is typically used to assign the electronic density associated with an atom within a molecule, and it yields noninteger values which do not correspond, in general, to the integer number of electrons assigned to subsystem $A$. Some renormalization procedures can be followed to correct this problem. Since they are arbitrary, we leave the problem of the partitioning of the density for a further analysis in a future work since the errors in the cases studied here involving identical atoms are small.

\section{B. Embedding potential inversion}

According to the DFT theory, ${ }^{71}$ the density determines the external potential that governs all the properties of the system. The inversion of such potential is a fundamental problem in DFT theory and has been the subject of an intense work. ${ }^{47-57}$ Here we simply establish a similarity between such an external field and the embedding potential searched in this work.

Let us assume an iterative procedure such that the density difference as $\Delta^{k}=\rho_{A}-\rho_{A}^{k}$ is minimized as the iteration number $k$ increases. $\rho_{A}^{k}$ is the density obtained from the modified HF equation, Eq. (1), at an iteration $k$. When $\Delta^{k}>0$, the potential would act as an "attractor" for the density to the region where there is a shortage. In contrast, for $\Delta^{k}<0$, a repulsive potential should be added to "remove" some density where there is too much. Thus, generalizing the DFT concepts, it can be concluded that the correction to the embedding potential is a functional of the density difference $\Delta^{k}$, i.e., $\mathcal{C}[\Delta]$. It can be considered that a potential matrix represented in an atomic basis set is sufficiently general to achieve the $\Delta^{k}=0$ condition. The number of terms, $n \times(n+1) / 2$, is however, too large to be adjusted. Some particular functional should then be employed. 
Here we use a modification of the ZMP (Ref. 50) procedure. For a given iteration $k$, the correction to the embedding potential that minimizes $\Delta^{k+1}$ is defined as

$$
\mathcal{C}^{k}\left[\lambda, \Delta^{k-1}\right]=\lambda \quad v^{k}\left[\Delta^{k-1}\right],
$$

with the self-repulsion potential being

$$
v^{k}\left[\Delta^{k-1}\right]=\int d \mathbf{r} \frac{\Delta^{k-1}}{\mathbf{r}-\mathbf{r}^{\prime}}
$$

Such potential corresponds to the electric field arising from the density difference $\Delta^{k}$ and introduces a driving force to make $\rho_{A}^{k}$ coincide with the reference $\rho_{A}$ one. This selfrepulsion term presents a parallelism with the energy localization procedure in which local orbitals are determined by minimizing the Coulomb and/or exchange energies, Eq. (18), between densities built with orbitals localized in different regions by simply replacing the terms depending on $\rho$ and $\rho^{B}$ by $\Delta$. In addition, the use of such potential presents the advantage of using bielectronic integrals, thus avoiding the evaluation of new integrals and simplifying the use of any $a b$ initio package. Some other $v_{\lambda}^{k}[\Delta]$ functionals could be designed to improve the final embedding potential as proposed by other authors, ${ }^{49,53,57,60}$ but this is left for future work. In Eq. (20), $\lambda$ is a parameter that will be fitted to minimize

$$
\delta^{k}=\int d \mathbf{r}\left(\Delta^{k}\right)^{2}=\operatorname{Tr}\left[\mathbf{S} \Delta^{\mathbf{k}} \mathbf{S} \Delta^{\mathbf{k}}\right]
$$

where $\mathbf{S}$ and $\boldsymbol{\Delta}^{k}$ are the overlap and density difference matrices represented in the atomic basis set, as defined by Tozer et $a l .{ }^{53}$ Since the density difference $\Delta^{k}$ can be positive and negative, it is convenient to work with its square as a measure of the real density difference. Such quantity could be, in principle, set to zero at convergence when using an infinite basis set, but in real applications it can only be minimized.

When the two densities coincide, the self-repulsion energy defined as

$$
E_{\mathrm{rep}}^{k}=\frac{1}{2} \iint d \mathbf{r} d \mathbf{r}^{\prime} \frac{\Delta^{k}(\mathbf{r}) \Delta^{k}\left(\mathbf{r}^{\prime}\right)}{\mathbf{r}-\mathbf{r}^{\prime}}
$$

has to be zero or at least minimized.

At a given iteration $k, \rho^{k}=\rho_{A}^{k}+\rho_{B}$ can be defined, so that a new energy $E_{\mathrm{DFT}}^{k}$ can be obtained using Eq. (11). Thus, at convergence, $E_{\mathrm{DFT}}-E_{\mathrm{DFT}}^{k}$ should be also minimized.

Thus the iterative procedure proposed here is as follows:

(1) For simplicity it can be considered that initially $\mathcal{V}^{0}=0$. In some situations, however, the convergence of the method could be accelerated by assuming an initial guess for $\mathcal{V}^{0}$. In particular, using different DFT functionals it would be convenient to choose the $V_{\mathrm{xc}}\left[\rho^{A B}\right]$ $-V_{\mathrm{xc}}\left[\rho^{A}\right]$. A calculation for the isolated $A$ subsystem using the modified Fock equations with this $\mathcal{V}^{0}$ is then performed to obtain $\rho_{A}^{0}$.

(2) With a given $\rho_{A}^{k}$ the self-repulsion potential $v^{k}\left[\Delta^{k-1}\right]$ is calculated, and the modified Fock equation

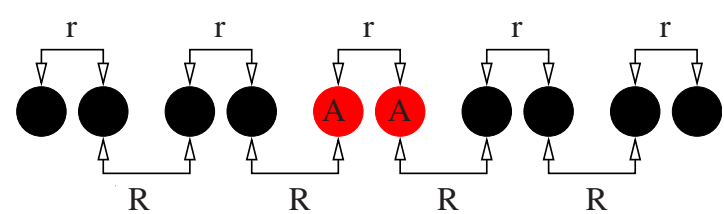

FIG. 2. (Color online) Nuclear geometry used to describe the $\left(\mathrm{H}_{2}\right)_{5}$ system. The two atoms in red with an $A$ symbol are those included in subsystem $A$.

$$
\left[\hat{\mathcal{F}}_{A}+\hat{\mathcal{V}}^{k-1}+\mu v^{k}\left[\Delta^{k-1}\right]\right] \phi_{l}^{k}=\epsilon_{l}^{k} \phi_{l}^{k}
$$

is solved for several $\mu$ values in a given range. The $\lambda$ value is determined as the one corresponding to the $\mu$ value that yields the lower $\delta^{k}$ in Eq. (22). With it, the correction to the embedding potential in Eq. (20) is completely determined, and the new embedding potential for the next iteration is obtained as

$$
\mathcal{V}^{k}=\mathcal{V}^{k-1}+\mathcal{C}^{k}\left[\lambda, \Delta^{k}\right]=\mathcal{V}^{k-1}+\lambda v^{k}\left[\Delta^{k}\right]
$$

In general, $\lambda$ can be defined as a matrix in the atomic basis set representation as $\mathcal{V}$. In this particular implementation, however, $\lambda$ is a simple number multiplying the identity matrix.

(3) Adding $\mathcal{V}^{k}$ to the Fock operator corresponding to subsystem $A$, the modified HF equation

$$
\left[\hat{\mathcal{F}}_{A}+\hat{\mathcal{V}}^{k}\right] \phi_{l}^{k}=\epsilon_{l}^{k} \phi_{l}^{k}
$$

is solved and the new $\rho_{A}^{k+1}$ is obtained.

(4) With the updated $\rho_{A}^{k+1}$, the new density difference $\Delta^{k+1}$ is obtained, and if $\delta^{k+1}$ is larger than a given threshold $\epsilon$, the iteration is started again at point (2). Once $\delta^{k}<\epsilon$ or the number of iterations exceeds a given maximum number, the iterative procedure is stopped. $\Delta^{k}=0$ is, however, not reached, in general. First, it is not warranted that the reference density $\rho_{A}$ is $v$ representable, and some other terms could be necessary. Second, the repulsion potential functional form is not sufficiently flexible, in general, to achieve $\Delta^{k}=0$, and hence the self-repulsion potential $v^{k}\left[\Delta^{k}\right]$ does not vanish either. Thus, the iterative procedure is finished when the $|\lambda|<\epsilon$ condition is satisfied.

\section{Correlated ab initio calculations and total correlated energy}

With the $\mathcal{V}$ potential thus determined, the resolution of the modified HF equation is performed to obtain the molecular orbitals, the density $\rho_{A}^{k}$, and the HF energy $E_{\mathrm{HF}}^{A}$. Later on, a MCSCF calculation is performed to reoptimize the orbitals and include some correlation. Finally, a MRCI calculation is carried out to obtain the electronic energies of the ground and excited levels $E^{A, C I}$.

For the ground electronic state, the correlation energy is determined as $E_{\mathrm{cor}}^{i=0}=E_{A, \mathrm{CI}}^{i=0}-E_{\mathrm{HF}, A}$. The energy of the total system $A B$, including correlation, for the ground state is then given by 


$$
E_{\mathrm{CI}}^{0}=E_{\mathrm{HF}}^{\mathrm{tot}}+E_{\mathrm{cor}}^{i=0}=E_{\mathrm{tot}, \mathrm{HF}}+E_{A, \mathrm{CI}}^{i=0}-E_{\mathrm{HF}, A},
$$

where $E_{\mathrm{tot}, \mathrm{HF}}$ is the HF energy obtained for the whole system $A B$, i.e., the DFT correlation energy is not included to avoid double counting of this term.

For the excited electronic states the energy difference, including correlation, is obtained as $E_{\mathrm{dif}}^{i}=E_{A, \mathrm{CI}}^{i}-E_{\mathrm{HF}, A}$ (with $E_{\mathrm{HF}, A}$ corresponding to the ground HF energy), so that the correlated energy becomes

$$
E_{\mathrm{CI}}^{i}=E_{\mathrm{tot}, \mathrm{HF}}+E_{\mathrm{dif}}^{i}=E_{\mathrm{tot}, \mathrm{HF}}+E_{A, \mathrm{CI}}^{i}-E_{\mathrm{HF}, A} .
$$

\section{RESULTS AND DISCUSSION}

The results presented in this work correspond to systems with different numbers of hydrogen atoms, $\left(\mathrm{H}_{2}\right)_{5}$ and $\mathrm{H}_{2}+\left(\mathrm{H}_{2}\right)_{5}$, taken as benchmark systems to analyze the ability of the method to describe delocalization and van der Waals interactions. The hydrogen atoms are described by the VTZ basis of Dunning, $\mathrm{Jr}^{81}$ including 16 functions, $s, p$, and $d$. Exact calculations on the entire systems were performed to obtain benchmark results. Thus, an initial HF calculation were followed by a MCSCF calculation to optimize the molecular orbitals and select the reference electronic configurations finally used in the multireference single and double configuration interaction (CI) calculation. The MCSCF calculations are performed including a full valence active space, considering 10 and 12 active orbitals for $\left(\mathrm{H}_{2}\right)_{5}$ and $\mathrm{H}_{2}+\left(\mathrm{H}_{2}\right)_{5}$, respectively. The MRCI calculations are done using the internally contracted MRCI method ${ }^{82,83}$ implemented in the MOLPRO package. ${ }^{84}$ The reference configurations are automatically chosen as those optimized in the previous MCSCF, being of the order of 20000 and 75000 for $\left(\mathrm{H}_{2}\right)_{5}$ and $\mathrm{H}_{2}+\left(\mathrm{H}_{2}\right)_{5}$, respectively. Thus, the numbers of internally contracted configurations used in the CI calculations are approximately $5 \times 10^{6}$ and $60 \times 10^{6}$, respectively. The calculations for the $A$ subsystem are performed using the basis set of the entire system but setting the charges in the nuclei outside $A$ to zero. The method used for the correlated energies on clusters $i$ are essentially the same as those performed on the whole systems but restricted to a lower number of electrons. The method has been implemented in the MOLPRO package of programs ${ }^{84}$ using the MATROP utilities.

\section{A. $\left(\mathrm{H}_{2}\right)_{5}$ chain}

To show how the method works, it is first applied to $\left(\mathrm{H}_{2}\right)_{5}$ in the linear configuration shown in Fig. 2 with $r=R$ $=1.3$ a.u. The HF density of the total system, $\rho$, is displayed in the bottom panel of Fig. 3. The total electronic density is rather delocalized, but there are also important boundary conditions introduced by the small number of atoms which explains why the densities around different atoms are not exactly equivalent. The density of the $A$ subsystem, $\rho_{A}$, obtained using the Mulliken-type partition from Eq. (19) and displayed in the top panel of Fig. 3, is rather different in between the two hydrogen atoms. This is probably due to the non-negligible contribution of basis functions placed on other atoms.

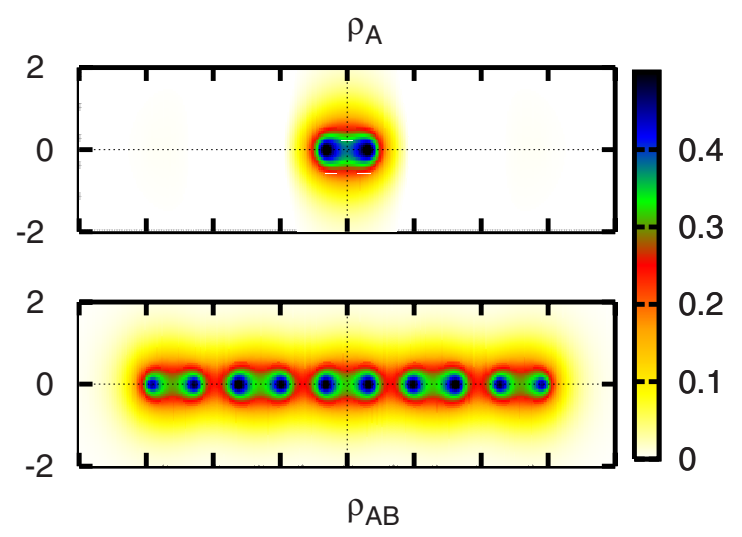

FIG. 3. (Color online) Total density of $\left(\mathrm{H}_{2}\right)_{5}$ obtained at the HF level with $r=R=1.3$ a.u. (bottom panel) and density of the $A$ subsystem extracted using the Mulliken-type partition method (top panel).

The iterative procedure proposed tends to reduce the density difference $\Delta^{k}$, as it is shown in Fig. 4 starting from $k=0$, with no embedding potential and just using the Fock operator of one $\mathrm{H}_{2}$ subunit, up to $k=5$ at which the minimization yields $\lambda=0$. Initially the larger density difference is around the two nuclei of the $A$ cluster, where it is too low, and their closest neighbors, where it is too high. The density difference in the $A$ subsystem decreases significantly in the iterative procedure, while the improvement is insignificant in the edges of the cluster. Once the difference cannot be reduced anymore, at $k=5$, some oscillations whose zeros correspond to the actual positions of the nuclei (where the elec-

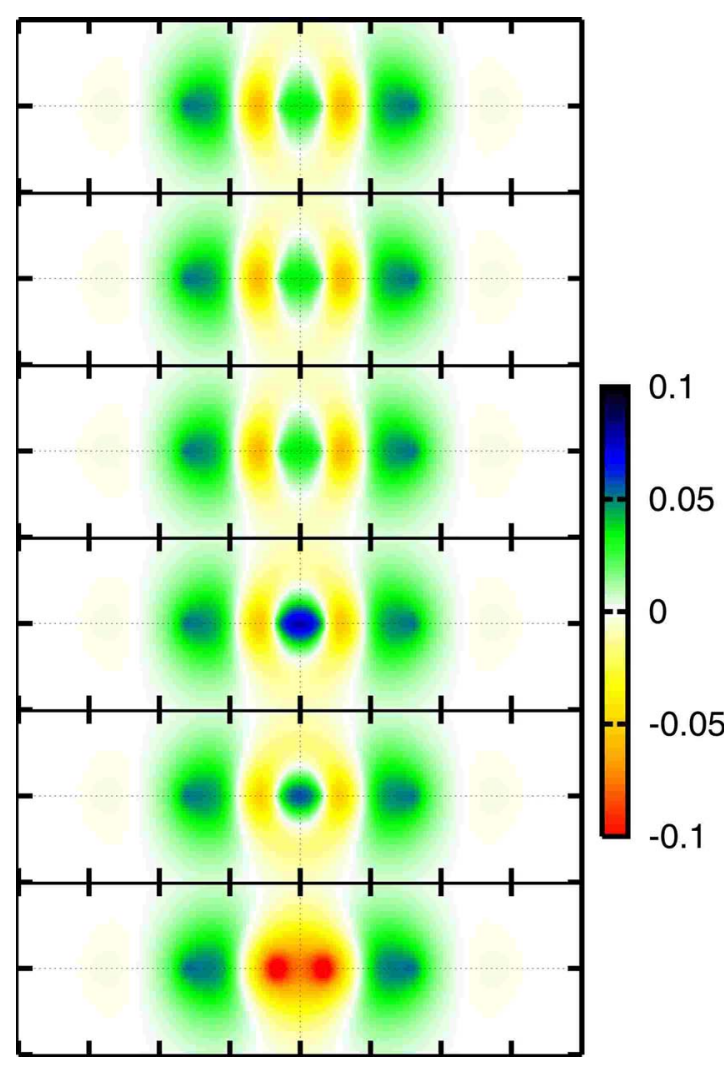

FIG. 4. (Color online) Density difference $\Delta^{k}$ obtained for $\left(\mathrm{H}_{2}\right)_{5}$ in the $X Z$ plane as a function of the iteration from $k=0$ (bottom panel), corresponding to no embedding potential, up to $k=5$ (top panel), from where no more reduction in the integral of the square of the density difference is found. 


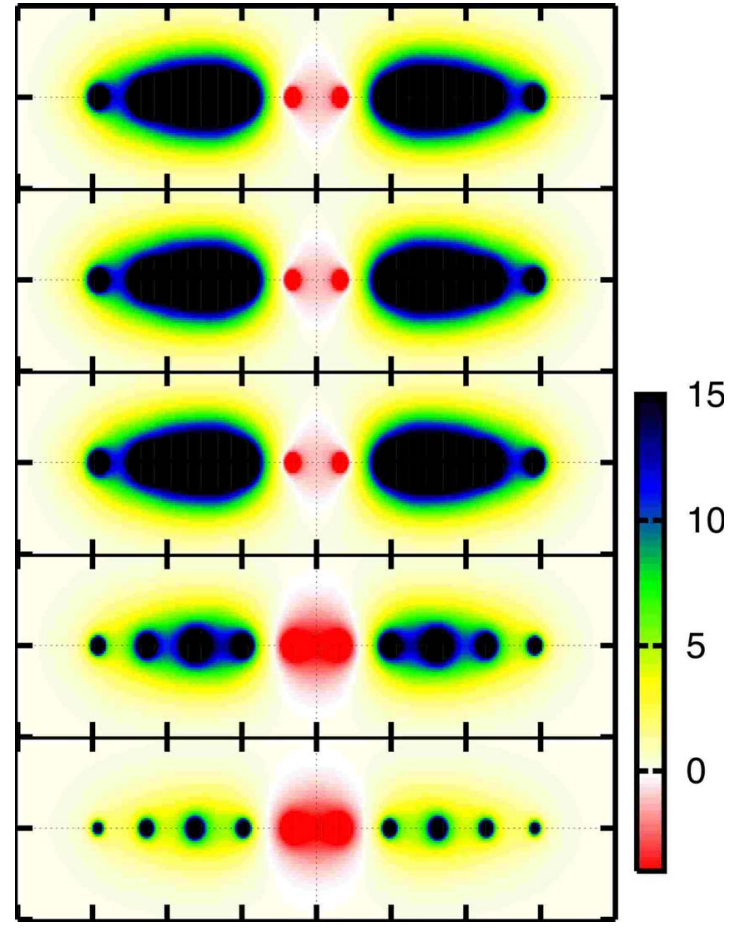

FIG. 5. (Color online) Embedding potential $\mathcal{V}^{k}$ obtained for $\left(\mathrm{H}_{2}\right)_{5}$ as a function of the iteration from $k=1$ (bottom panel) up to $k=5$ (top panel). Energies and distances are in a.u.

tronic basis is richer and can yield to a better description) appear. In fact, the description can be improved by increasing the basis set. If the overlap among the functions becomes too large, however, other numerical problems arise. This is why bond functions are not very useful in solving this problem.

The lack of convergence in the density difference may be due to two major reasons. First, the self-repulsion potential is not flexible enough, and some new functionals of the density difference should be investigated. Second, the partition of the total density introduces an artificial sudden cutoff at the boundaries, which might be responsible for the oscillatory behavior of $\Delta^{k}$, somehow reminding the aliasing using Fourier transforms. ${ }^{85}$ More refined methods to partition the density should be tried to analyze if such effects could be eliminated.

The embedding potential, in Fig. 5, shows a fast convergence with the iterations, being rather stable for $k=4$. It is negative in subsystem $A$ to increase the density in that region, while it is rather repulsive outside $A$. This form localizes the density in the $A$ subsystem and allows obtaining molecular orbitals localized in $A$. Of course, such localized orbitals could be obtained for the isolated $A$ subsystem as well. The important difference is that the embedded orbitals together with the modified Fock operator through the inclusion of the embedding potential allow the effective description of the interaction of subsystem $A$ with its environment, doing calculations only on subsystem $A$.

This is illustrated in Fig. 6 where the energy curves obtained for the whole system from DFT, MCSCF, and MRCI calculations (full symbols denoted as $A B$ ) are compared with those obtained with the present method using the embedding

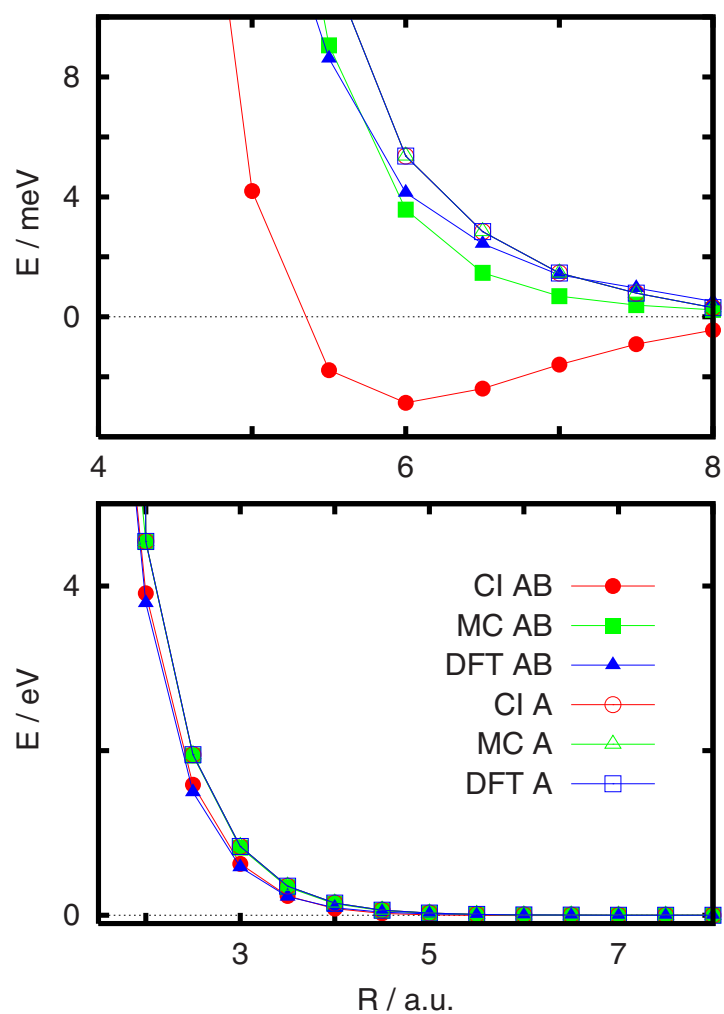

FIG. 6. (Color online) Energy curves obtained for $\left(\mathrm{H}_{2}\right)_{5}$ as a function of the $R$ distance, setting $r=1.3$ a.u. Full symbols correspond to calculations performed for the entire system (16 electrons) with DFT, MCSCF, and MRCI methods, while open symbols correspond to the same type of calculations but only on subsystem $A$ (only 2 electrons). Top panel corresponds to an inset to show the details. Distances are in a.u. and energies in eV. Each curve has been shifted to its own asymptotic value obtained for $r=1.3$. a.u. and $R=9$. a.u.

potential and only the electrons of subsystem $A$ (empty symbols denoted as $A$ ). Concerning the calculations on the whole system, they show an interaction that is essentially repulsive, with similar slope for nearly all the cases. Only the MRCI-AB calculation yields a shallow van der Waals well, of $\approx 3 \mathrm{meV}$, since it is the only method that describes properly the dynamic correlation.

The different calculations within the embedding method developed here only treats two electrons corresponding to the $A$ subsystem, composed by the two central hydrogen atoms. It is notorious that using the embedding potential, the three methods are able to reproduce rather well the repulsive effect introduced by neighboring atoms, showing its ability to provide a reasonably good effective Hamiltonian, which accounts for "solvation" effects. The variation with the distance of the energies obtained with the three methods using the embedding potential, i.e., DFT-A, MC-A, and CI-A, are nearly indistinguishable. Electronic correlation does not introduce any difference. This indicates that the repulsive barrier is entirely described by the embedding potential. Moreover, the good agreement of the embedding results with those obtained describing the whole system indicates that the repulsive barrier is well described by an average.

In this case, it is obvious that the MRCI-A cannot reproduce dynamic correlation effects between cluster $A$ and the neighboring atoms because the excitations of these last at- 


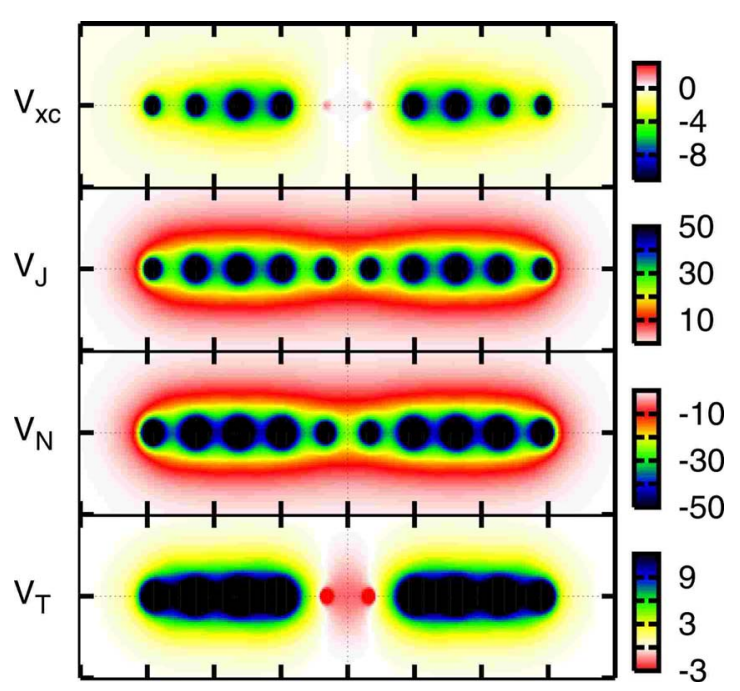

FIG. 7. (Color online) Components of the embedding potential arising in Wesolowski and Warshell (Ref. 32) method, Eq. (14), but with $\rho^{A}$ being obtained using the Mulliken-type density partition method: $V_{T}$ is the kinetic contribution using a Thomas-Fermi functional, $V_{N}$ is the nuclear attraction term, $V_{J}$ is the Coulomb term, and $V_{\mathrm{xc}}$ is the exchange-correlation term using a PW91 DFT functional. Energies are in a.u.

oms are not considered. This is why MRCI-A and MCSCF-A results are nearly indistinguishable. Obviously, the only way to reproduce such van der Waals interaction is via the inclusion of the atoms more directly involved, as described below for the $\mathrm{H}_{2}+\mathrm{H}_{10}$ system.

\section{B. Comparison with DFT embedding potential}

Let us analyze the physical meaning of the different embedding potential terms arising from the method developed by Wesolowski and Warshel, ${ }^{32}$ Eq. (14), as used in Carter and co-workers's ${ }^{42-44}$ method. These are shown in Fig. 7 using the density $\rho_{A}$ obtained from the Mulliken-type partition expressed in Eq. (19) and also employed in Sec. IV A. The kinetic energy term $\mathcal{V}_{T}$, using a Fermi functional, shows a topology very close to that of the total embedding potential obtained with the present method in Fig. 5: it is slightly attractive in the region of cluster $A$, while it is repulsive out of it, thus localizing the density in region $A$ (as discussed in Sec. IV A). The nuclear attraction term $\mathcal{V}_{N}$ is more attractive outside region $A$ since it corresponds to the nuclear attraction terms from the nuclei placed in region $B$. The clear minima around the nuclei are due to the use of a discretized basis set centered at the nuclei, which clearly yields to a rather good description at the position of nuclei, while it is poorer in between them. The Coulomb term, $\mathcal{V}_{J}=2 \mathbf{J}^{A B}-2 \mathbf{J}^{A}$, provides the repulsion due to the electronic density of the $B$ subsystem and is everywhere positive. $\mathcal{V}_{J}$ is very similar to $-\mathcal{V}_{N}$, and when added it should provide a rather small contribution. The fourth term is the exchange-correlation term $\mathcal{V}_{\mathrm{xc}}$, which in this case is described using the Perdew-Wang PW91 functional and shows a similar structure to $\mathcal{V}_{T}$ but with opposite sign.

The $\mathcal{V}_{N}$ and $\mathcal{V}_{\mathrm{xc}}$ contributions are more attractive in region $B$ than in region $A$. Thus, they contribute to the delocalization of the density out of the region of cluster $A$. The contributions that localize the density on $A$ are $\mathcal{V}_{T}$ and $\mathcal{V}_{J}$.
Since $\mathcal{V}_{J}$ is canceled by $\mathcal{V}_{N}$, the kinetic energy contribution $\mathcal{V}_{T}$ to the embedding potential is crucial in obtain a good embedding potential. The $\mathcal{V}_{T}$ and $\mathcal{V}_{\mathrm{xc}}$ contributions also somehow cancel each other. Therefore, it is important to choose the precise kinetic and exchange-correlation energy functionals in a balanced way

The $\mathcal{V}_{T}, \mathcal{V}_{N}$, and $\mathcal{V}_{N}$ potentials depend on the density $\rho^{A}$. Thus, when summing the four contributions, with the $\rho^{A}$ provided by the Mulliken-type partition method, the embedding potential does not present an appreciable barrier separating cluster $i$ from the rest of the system. In Carter and co-workers's ${ }^{42-44}$ method, however, $\rho^{A}$ is updated iteratively and it is expected that the embedding potential obtained with the converged density fulfills localization requirements similar to those found here.

This analysis shows the importance of the kinetic energy terms in the embedding potential. It also shows that the two terms better suited to build the embedding potential are $\mathcal{V}_{T}$ and $\mathcal{V}_{J}$. The election of $\mathcal{V}_{T}$ presents the problem of the arbitrariness of choosing a given functional of the density. This is the reason why the election of $\mathcal{V}_{J}$ is very well suited, as proposed by Zhao et al. ${ }^{50}$

\section{C. van der Waals interactions of $\mathbf{H}_{2}$ with a $\mathbf{H}_{10}$ chain}

We shall consider a $\mathrm{H}_{2}$ molecule (frozen at an internuclear distance of 1.3 a.u.) interacting with a $\mathrm{H}_{10}$ chain, with a distance between adjacent hydrogen atoms of 1.3 a.u. The $\mathrm{H}_{10}$ is placed parallel to the $z$ axis with the center of mass at the origin. The $\mathrm{H}_{2}$ is placed parallel to the $y$ axis with the center of mass along the $x$ axis at a distance $R$, which is varied.

In the embedding method, the DFT density obtained for the whole system $A B$ is partitioned, as $\rho^{A}+\rho^{B}$, considering a cluster $A$ formed by the two central atoms in the $\mathrm{H}_{10}$ chain and the isolated $\mathrm{H}_{2}$ molecule, thus including only four electrons. With $\rho^{A}$, the embedding potential $\mathcal{V}$ is obtained iteratively for each $R$ distance, where the $\lambda$ parameter is optimized in the $[-20,0]$ interval. For describing the weak van der Waals interaction, dynamical correlation needs to be included and for this reason only the most accurate CI results will be discussed. Present DFT-A results underestimate this well while MC-A results overestimate it. The CI-A energies obtained for different number of iterations are shown in Fig. 8 to show the convergency of the procedure. The number of iterations needed varies with the distance, being 3 over the whole interval. At short internuclear distances, some new iterations are added, with a $\lambda$ parameter inside the interval considered, whose evolution with $R$ is also shown in Fig. 8. It is found that it is convenient to keep $\lambda$ negative to maintain the stability of the convergence.

At each iteration, each contribution of the embedding potential adds a small change in total energy (of the order of $10^{-5}$ a.u. for $R=9$ ), as correlation potentials do in DFT calculations, which is quite significant dealing with so tiny wells. A contribution of this energy change produces a parallel shift of the whole curve, which can be eliminated by setting to zero the energy at long distances as done in Fig. 8, for the first three iterations. For long distances a higher num- 

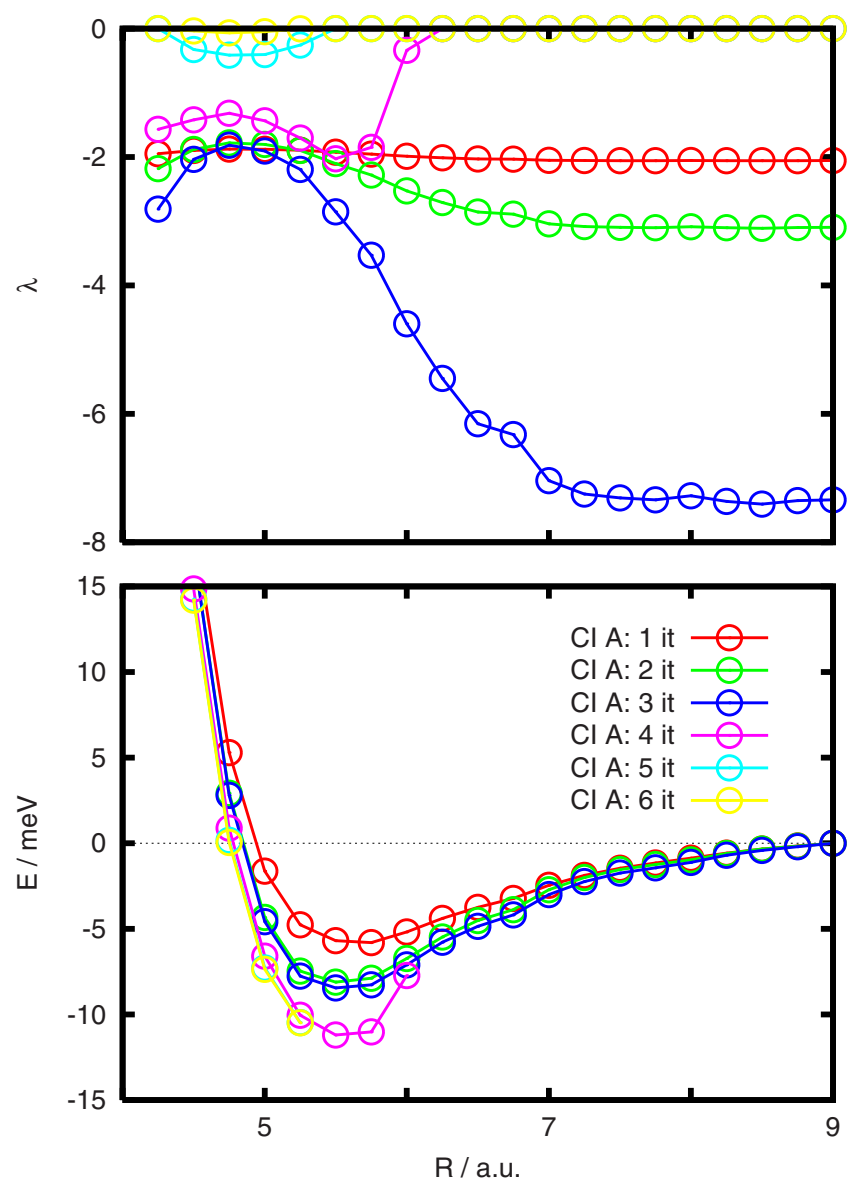

FIG. 8. (Color online) Bottom panel: Convergency of the energy curves obtained for $\mathrm{H}_{2}+\mathrm{H}_{10}$ for different numbers of iterations in the embedding procedure. Only CI calculations are shown. $R$ is the distance between the center of mass of $\mathrm{H}_{10}$ (along the $z$ axis with a unique internuclear distance $r=1.3$. a.u.) and the center of mass of $\mathrm{H}_{2}$, placed parallel to the $y$ axis at a distance $R$ along the $x$ axis (with an internuclear distance of also 1.3 a.u.). Top panel: optimum $\lambda$ obtained for each iteration as a function of $R$.

ber of iterations is not found with the present convergence criteria, essentially reflecting the lack of flexibility beyond this point of the self-repulsion potential chosen, in Eq. (21), and to the incompleteness of the basis set to represent the embedding potential. As a consequence, the asymptotic energy of iteration 3 is subtracted to the curves corresponding to a higher number of iterations. This is the reason why the energy for the case of four iterations shows a sudden jump down at $R=5.75$ a.u., just when $\lambda_{4}$ becomes significant. Since the progression from one to three iterations shows a gradual convergence of the energy curves, here we will keep as the best result the curve obtained for three iterations to avoid artificial jumps.

Note that a different degree of convergence of the embedding potential can introduce also artificial relative energy shifts among adjacent points, similar to the usual basis set superposition errors, which are not evaluated here to simplify and because all MRCI calculations are done using the same basis set, being then comparable. To avoid this problem, especially for so tiny binding energies, it is important to determine the optimum $\lambda$ with enough accuracy to obtain smooth curves.

The embedding procedure described is restricted to sub-
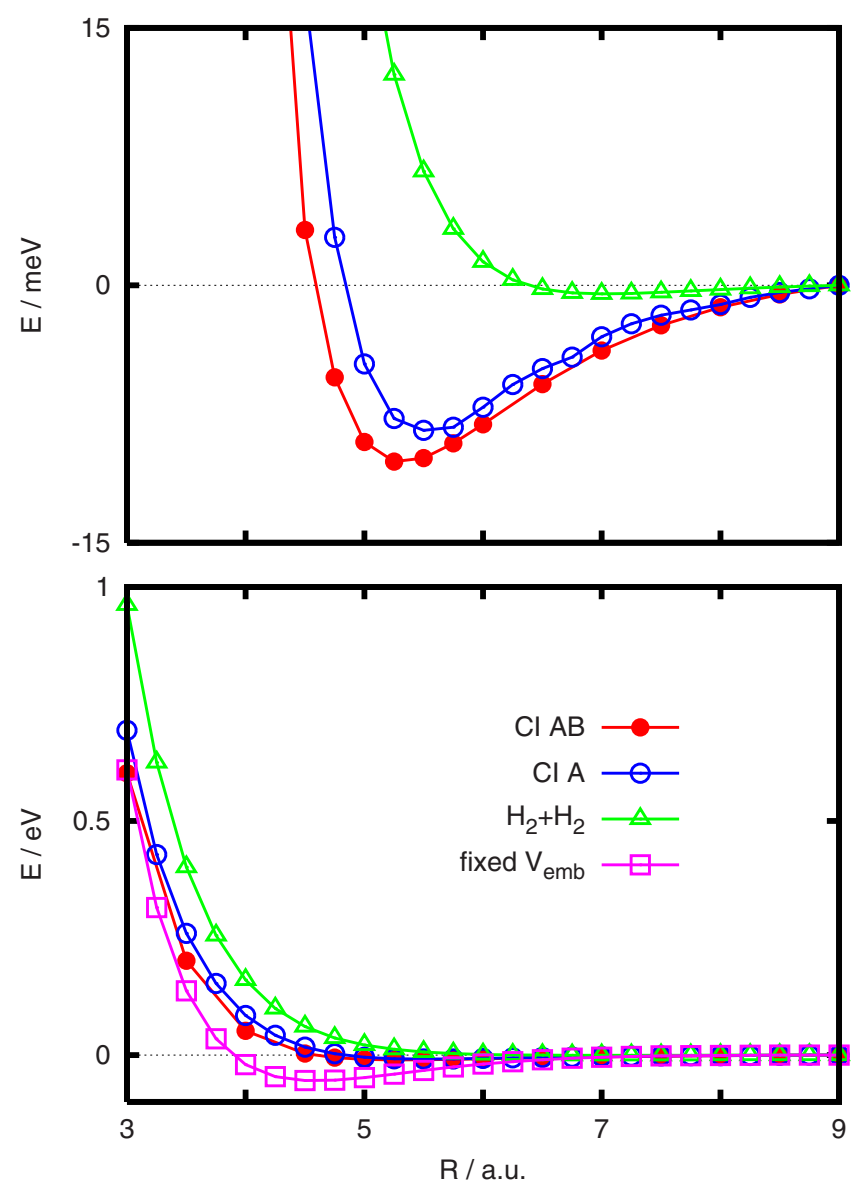

FIG. 9. (Color online) Comparison of the energies obtained using different methods for $\mathrm{H}_{2}+\mathrm{H}_{10}$. The geometries are the as described for Fig. 8. Full circles (CI-AB) correspond to a MRCI calculation on the whole system. Open circles (CI-A) are the MRCI energies obtained using an embedding potential using three iterations, where the active region corresponds to the isolated $\mathrm{H}_{2}$ and the two central $\mathrm{H}$ atoms of the $\mathrm{H}_{10}$ chain. Open squares (fixed $\mathcal{V}_{\text {emb }}$ ) correspond to MRCI calculation using a unique embedding potential, obtained for $\mathrm{H}_{10}$ as described in Sec. IV A. Open triangles $\left(\mathrm{H}_{2}+\mathrm{H}_{2}\right)$ correspond to MRCI calculations of the $\mathrm{H}_{2}-\mathrm{H}_{2}$ subsystem including the basis of the whole system, i.e., is equivalent to $\mathrm{CI}-\mathrm{A}$ but without an embedding potential.

system $A$ including only four electrons and using the Fock equation modified by $\mathcal{V}$. To check the accuracy of the procedure, MRCI calculations on the whole system are also performed, which treats 12 electrons, yielding more difficult calculations. The corresponding energy, CI-AB, in Fig. 9, presents a shallow well of $10 \mathrm{meV}$, while in the embedding procedure, CI-A, it is of $8.5 \mathrm{meV}$, at slightly longer distances. Such nice agreement shows the adequacy of the procedure proposed. To improve the accuracy a more flexible potential function should then be considered.

To obtain continuous potentials reducing the computing time, a second alternative considered is to use a single embedding potential $\mathcal{V}$ for all $R$ values, corresponding to the isolated $\mathrm{H}_{10}$ chain, described in Sec. IV C. The results thus obtained, denoted as "fixed $\mathcal{V}_{\text {emb" }}$ " in Fig. 9, clearly overestimates the van der Waals well, thus showing that this single $\mathcal{V}$ it is not flexible enough to describe the van der Waals interaction.

To quantify the improvement introduced by the $\mathcal{V}$ potential in the present method, a calculation on cluster $A$ is per- 
formed but without an embedding potential, denoted by $\mathrm{H}_{2}+\mathrm{H}_{2}$ in Fig. 9. Such calculation corresponds to that of the isolated $\left(\mathrm{H}_{2}\right)_{2}$ dimer but using the basis set of the 12 atoms, composed by 182 atomic functions. This calculation can then be considered as iteration 0 in the progression shown in Fig. 8 for the embedding procedure. The van der Waals well found is by far much weaker, of only $\approx 0.5 \mathrm{meV}$, and shifted to a much longer distance, $\approx 7$ a.u.

By careful inspection of Fig. 9 it is found that the embedding potential technique proposed in this work is accurate enough to fairly reproduce the van der Waals well of the $\mathrm{H}_{2}+\mathrm{H}_{10}$ system, with an effort comparable to that inverted in the calculation of the $\left(\mathrm{H}_{2}\right)_{2}$ dimer potential. This method can be viewed as the construction of an effective Fock equation, giving rise to localized molecular orbitals, which are very well suited for treating correlation effects within a predetermined subsystem $A$. The results obtained demonstrate the high power of such embedding methods to treat van der Waals interactions.

\section{CONCLUSIONS}

In this work, a new procedure to obtain an embedding potential to build a modified Fock equation and treat the local electronic correlation in subsystem $A$ of interest is proposed. Such method consists in, first, partitioning the density of the whole system, separating the one corresponding to subsystem $A$. The total density could be obtained at any level of accuracy depending on the size of the system. In the second step, the embedding potential is obtained iteratively using as a driving force the self-repulsion introduced by the density difference, in a similar form as proposed by Zhao et $a l{ }^{50}$ to obtain the exchange-correlation potential from a given exact density used as a reference. Finally, the electronic correlation can be treated in subsystem $A$ with any currently available method, such as MCSCF, CASPT2, or MRCI, using the molecular orbitals localized in subsystem $A$ by the embedding potential so obtained.

The role played by the kinetic term in embedding methods is analyzed and it is found to be crucial since it creates barriers separating subsystem $A$ from the environment. Such "localization" barriers localize the molecular orbitals and thus allow a local treatment of the electronic correlation in subsystem $A$. In DFT-based embedding methods, ${ }^{42-44}$ the kinetic term is considered by using different functionals. The method presented here is designed as an alternative to such embedding DFT-based methods but avoiding the problems associated with the election of a given functional to treat the kinetic energy term.

The method has been first applied to a $\mathrm{H}_{10}$ linear chain for an internuclear distance of 1.3 a.u., where the system presents a quite delocalized density. The iterative procedure is illustrated and the physical meaning of the potential is discussed by comparing with the different terms appearing in Wesolowski and Warshell ${ }^{32}$ embedding potential, focusing again on the kinetic energy contribution.

When applied to $\mathrm{H}_{10}$ varying the distance among the atoms, the method shows that the description of the central
$\mathrm{H}_{2}$ with an embedding potential is able to describe the repulsive potential interaction with the environment, reducing enormously the computation time.

Finally, the method is applied to study the van der Waals interactions between a $\mathrm{H}_{10}$ chain and a $\mathrm{H}_{2}$ molecule. A cluster formed by the central $\mathrm{H}_{2}$ subunit in the $\mathrm{H}_{10}$ chain and the approaching $\mathrm{H}_{2}$ molecule is considered explicitly, thus reducing considerably the number of electrons considered in the calculations, and hence the computational cost. The comparison of the results with exact MRCI calculations on the whole system shows very good agreement. In particular, the position and well depth obtained with the embedding method are rather satisfactory, with an error of only 1-2 meV for a total well depth value of about $8 \mathrm{meV}$. This good agreement proves the ability of the method to describe such weak interactions.

Two major weakness of the method are found, which need to be improved: the density partitioning procedure and the form of the self-repulsion potential used. Concerning the first problem, several alternatives to the Mulliken-like partitioning were considered to correct the noninteger number of electrons in subsystem $A$. However, the changes produced in the interaction potentials in the systems under study were small. For $\left(\mathrm{H}_{2}\right)_{5}$, as the distance between monomers decreases, the charge difference increases. In this case, the repulsive character of the potential arises when the electronic clouds around each $\mathrm{H}_{2}$ monomer overlap. Such repulsion effect is reasonably well reproduced by the embedding potential giving rise to potential interaction results in rather good agreement with the exact results. The density partitioning made for the $\mathrm{H}_{2}+\left(\mathrm{H}_{2}\right)_{5}$ system is essentially equivalent for all distances considered because the division affects the $\left(\mathrm{H}_{2}\right)_{5}$ monomer which is kept fixed. This explains why the different renormalization alternatives considered produced similar results, differing by only $1-2 \mathrm{meV}$ for the dissociation energy. The problems associated with the partitioning using this method are reduced by increasing the size of cluster $A$, as in the buffered divide-and-conquer techniques, ${ }^{27-29}$ but the computational effort would increase significantly. Therefore, new partition techniques should be investigated to improve the accuracy of the method. The second possibility of increasing the accuracy of the method is to consider new functionals for describing the self-repulsion potential terms, as those previously used by several authors $49,53,57,60$ to increase its flexibility. Apart from these two possibilities to improve the accuracy and performance of the method, the results presented here are rather encouraging and nowadays some further application to study the reactivity on different metallic nanostructures and van der Waals wells on larger systems are being developed.

\section{ACKNOWLEDGMENTS}

We thank Dr. D. R. Alcoba and Dr. A. O. Mitrushchenkov for very fruitful discussions. This work has been supported by Comunidad Autónoma de Madrid (CAM) under Grant No. S-0505/MAT/0303 and by the Ministerio de Ciencia e Innovación under Project Nos. CTQ2007-62898, FIS2007-62006, CTQ2007-63332, and MAT2007-60966. 
M.P.L.C. acknowledges the support by MEC-CSIC Project No. 2007501004

${ }^{1}$ P. Nieto, E. Pijper, D. Barredo, G. Laurent, R. A. Olsen, E.-J. Baerends, G.-J. Kroes, and D. Farías, Science 312, 86 (2006).

${ }^{2}$ Y. Wang and X. G. Gong, J. Chem. Phys. 125, 124703 (2006).

${ }^{3}$ L. Barrio, P. Liu, J. A. Rodriguez, J. M. Campos-Martin, and J. L. G. Fierro, J. Chem. Phys. 125, 164715 (2006).

${ }^{4}$ G. Hetzer, M. Schütz, H. Stoll, and H.-J. Werner, J. Chem. Phys. 113, 9443 (2000)

${ }^{5}$ M. Schütz and H.-J. Werner, J. Chem. Phys. 114, 661 (2001).

${ }^{6}$ H. Stoll, Phys. Rev. B 46, 6700 (1992).

${ }^{7}$ H. Stoll, J. Chem. Phys. 97, 8449 (1992).

${ }^{8}$ E. Voloshina, N. Gaston, and B. Paulus, J. Chem. Phys. 126, 134115 (2007).

${ }^{9}$ B. Paulus, Phys. Rep. 428, 1 (2006).

${ }^{10}$ C. Edmiston and K. Ruedenberg, Rev. Mod. Phys. 35, 457 (1963).

${ }^{11}$ J. Pipek and P. G. Mezey, J. Chem. Phys. 90, 4916 (1989).

${ }^{12}$ F. Aquilante, T. B. Pedersen, A. Sánchez-de-Merás, and H. Koch, J. Chem. Phys. 125, 174101 (2006).

${ }^{13}$ G. H. Wannier, Phys. Rev. 52, 191 (1937).

${ }^{14}$ S. Casassa, C. M. Zicovich-Wilson, and C. Pisani, Theor. Chem. Acc. 116, 726 (2006).

${ }^{15}$ R. F. W. Bader, Atoms in Molecules: A Quantum Theory (Oxford University Press, Oxford, UK, 1990).

${ }^{16}$ R. G. Gordon and Y. S. Kim, J. Chem. Phys. 56, 3122 (1972).

${ }^{17}$ M. P. Guse, J. Chem. Phys. 75, 828 (1981).

${ }^{18}$ L. Li and R. G. Parr, J. Chem. Phys. 84, 1704 (1986).

${ }^{19}$ W. Yang, Phys. Rev. Lett. 66, 1438 (1991).

${ }^{20}$ W. Yang and T.-S. Lee, J. Chem. Phys. 103, 5674 (1995).

${ }^{21}$ M. S. Daw and M. I. Baskes, Phys. Rev. B 29, 6443 (1984).

${ }^{22}$ P. Cortona, Phys. Rev. B 44, 8454 (1991).

${ }^{23}$ P. Cortona, Phys. Rev. B 46, 2008 (1992).

${ }^{24}$ T. Zhu, W. Pan, and W. Yang, Phys. Rev. B 53, 12713 (1996).

${ }^{25}$ A. J. Coleman, Rev. Mod. Phys. 35, 668 (1963).

${ }^{26}$ C. Valdemoro, L. M. Tel, and E. Pérez-Romero, Phys. Rev. A 61, 032507 (2000).

${ }^{27}$ B. G. Janesko and D. Yaron, J. Chem. Phys. 119, 1320 (2003).

${ }^{28}$ B. Bories, S. Evangelisti, T. Leininger, and D. Maynau, Chem. Phys. Lett. 385, 225 (2004).

${ }^{29}$ B. Bories, S. Evangelisti, D. Maynau, and T. Leininger, Theor. Chem. Acc. 116, 462 (2006).

${ }^{30}$ D. A. Mazziotti, Phys. Rev. E 65, 026704 (2002).

${ }^{31}$ D. R. Alcoba, Adv. Chem. Phys. 134, 205 (2007).

${ }^{32}$ T. A. Wesolowski and A. Warshel, J. Phys. Chem. 97, 8050 (1993).

${ }^{33}$ P. Nachtigall, K. D. Jordan, and K. C. Janda, J. Chem. Phys. 95, 8652 (1991).

${ }^{34}$ C. J. Wu and E. A. Carter, Phys. Rev. B 45, 9065 (1992).

${ }^{35}$ R. Konecny and D. J. Doren, J. Chem. Phys. 106, 2426 (1997).

${ }^{36}$ M. P. de Lara-Castells, A. O. Mitrushenkov, O. Roncero, and J. L. Krause, Isr. J. Chem. 45, 59 (2005).

${ }^{37}$ N. W. Winter, R. M. Pitzer, and D. K. Temple, J. Chem. Phys. 87, 2945 (1987).

${ }^{38}$ J. L. Pascual, L. Seijo, and Z. Barandiarán, J. Chem. Phys. 98, 9715 (1993).

${ }^{39}$ V. Puchin, A. Shluger, Y. Nakai, and N. Itoh, Phys. Rev. B 49, 11364 (1994).

${ }^{40}$ E. V. Stefanovich and T. N. Truong, J. Chem. Phys. 102, 5071 (1995).

${ }^{41}$ E. V. Stefanovich and T. N. Truong, J. Chem. Phys. 104, 2946 (1996).

${ }^{42}$ N. Govind, Y. A. Wang, and E. A. Carter, J. Chem. Phys. 110, 7677 (1999).

${ }^{43}$ T. Klüner, N. Govind, Y. A. Wang, and E. A. Carter, J. Chem. Phys. 116, $42(2002)$.
${ }^{44}$ P. Huang and E. A. Carter, J. Chem. Phys. 125, 084102 (2006).

${ }^{45}$ C. R. Jacob, S. M. Beyhan, and L. Visscher, J. Chem. Phys. 126, 234116 (2007).

${ }^{46}$ T. A. Wesolowski, J. Chem. Phys. 106, 7178 (1997).

${ }^{47}$ J. D. Talman and W. F. Shadwick, Phys. Rev. A 14, 36 (1976).

${ }^{48}$ C.-O. Almbladh and A. C. Pedroza, Phys. Rev. A 29, 2322 (1984).

${ }^{49}$ R. van Leeuwen and E. J. Baerends, Phys. Rev. A 49, 2421 (1994).

${ }^{50}$ Q. Zhao, R. C. Morrison, and R. G. Parr, Phys. Rev. A 50, 2138 (1994).

${ }^{51}$ O. V. Gritsenko, R. van Leeuwen, and E. J. Baerends, Phys. Rev. A 52, 1870 (1995)

${ }^{52}$ D. J. Tozer, V. E. Ingamells, and N. C. Handy, J. Chem. Phys. 105, 9200 (1996).

${ }^{53}$ D. J. Tozer, N. C. Handy, and P. Palmieri, Mol. Phys. 91, 567 (1997).

${ }^{54}$ G. K.-L. Chan and N. C. Handy, Phys. Rev. A 59, 2670 (1999).

${ }^{55}$ T. Gál, Phys. Rev. A 64, 062503 (2001).

${ }^{56}$ W. Yang and Q. Wu, Phys. Rev. Lett. 89, 143002 (2002).

${ }^{57}$ Q. Wu and W. Yang, J. Chem. Phys. 118, 2498 (2003).

${ }^{58}$ A. Görling and M. Levy, Phys. Rev. B 47, 13105 (1993).

${ }^{59}$ A. Görling and M. Levy, Phys. Rev. A 50, 196 (1994).

${ }^{60}$ T. Heaton-Burgess, A. J. Cohen, W. Yang, and E. R. Davidson, J. Chem. Phys. 128, 114702 (2008).

${ }^{61}$ A. F. Izmaylov, V. N. Staroverov, G. E. Scuseria, E. R. Davidson, G. Stoltz, and E. Cancés, J. Chem. Phys. 126, 084107 (2007).

${ }^{62}$ A. F. Izmaylov, V. N. Staroverov, G. E. Scuseria, and E. R. Davidson, J. Chem. Phys. 127, 084113 (2007).

${ }^{63}$ V. N. Staroverov, G. E. Scuseria, and E. R. Davidson, J. Chem. Phys. 124, 141103 (2006).

${ }^{64}$ V. N. Staroverov, G. E. Scuseria, and E. R. Davidson, J. Chem. Phys. 125, 081104 (2006).

${ }^{65}$ R. J. Bartlett, V. F. Lotrich, and I. V. Schweigert, J. Chem. Phys. 123, 062205 (2005).

${ }^{66}$ I. V. Schweigert, V. F. Lotrich, and R. J. Bartlett, J. Chem. Phys. 125, 104108 (2006).

${ }^{67}$ W. Kohn and L. J. Sham, Phys. Rev. 140, A1133 (1965).

${ }^{68}$ S. Baroni and E. Tuncel, J. Chem. Phys. 79, 6140 (1983).

${ }^{69}$ R. Colle and O. Salvetti, Theor. Chim. Acta 37, 785 (1987).

${ }^{70}$ F. A. Gianturco and M. P. de Lara-Castells, Int. J. Quantum Chem. 60, 593 (1996).

${ }^{71}$ R. G. Parr and W. Yang, Density-Functional Theory of Atoms and Molecules (Oxford Univesrity Press, New York, 1989).

${ }^{72}$ M. Dulak and T. A. Wesolowski, J. Chem. Theory Comput. 2, 1538 (2006).

${ }^{73}$ A. J. Stone, The Theory of Intermolecular Forces (Oxford Univerity Press, New York, 2002).

${ }^{74}$ D. R. Alcoba, A. Torre, R. C. Bochicchio, and L. Lain, Theor. Chem. Acc. 118, 827 (2007).

${ }^{75}$ J. F. Rico, R. López, G. Ramírez, I. Ema, and E. V. Ludeña, J. Comput. Chem. 25, 1355 (2004)

${ }^{76}$ T. E. Exner and P. G. Mezey, J. Phys. Chem. A 106, 11791 (2002).

${ }^{77}$ R. S. Mulliken, J. Chem. Phys. 23, 1833 (1955).

${ }^{78}$ J. F. Rico, R. López, and G. Ramírez, J. Chem. Phys. 110, 4213 (1999).

${ }^{79}$ D. R. Alcoba, L. Lain, A. Torre, and R. C. Bochichio, J. Chem. Phys. 123, 144113 (2005).

${ }^{80}$ D. R. Alcoba, R. C. Bochicchio, A. Torre, and L. Lain, J. Phys. Chem. 110, 9254 (2006).

${ }^{81}$ T. H. Dunning, Jr., J. Chem. Phys. 90, 1007 (1989).

${ }^{82}$ H.-J. Werner and P. J. Knowles, J. Chem. Phys. 89, 5803 (1988).

${ }^{83}$ P. J. Knowles and H.-J. Werner, Chem. Phys. Lett. 145, 514 (1988).

${ }^{84}$ MOLPRO, a package of ab initio programs designed by H.-J. Werner and P. J. Knowles, version 2006, with contributions from J. Almlöf, R. D. Amos, A. Berning et al.

${ }^{85}$ W. H. Press, S. A. Teukolsky, W. T. Vetterling, and B. P. Flannery, Numerical Recipes (Cambridge University Press, Cambridge, 1994). 